\title{
Editorial
}

\section{Ejection Fraction: Do You Know Your Number?}

\author{
MAtahar Ali
}

(Bangladesh Heart Journal 2017; 32(2) : 63-64)

Over the last 30 years, improvements in treatment and their implementation have improved survival and reduced the hospitalization rate in patients with heart failure (HF), although the outcome often remains unsatisfactory. The most recent European data (ESC-HF pilot study) demonstrated that 12-month all-cause mortality rates for hospitalized and stable/ambulatoryHF patients were $17 \%$ and $7 \%$ respectively and the 12 -month hospitalization rates were $44 \%$ and $32 \%$ respectively. The prevalence of this disease is approximately $1-2 \%$ IN adult population in developed countries. The life time risk of HF at age 55 is $33 \%$ for men and $28 \%$ for women.

The main terminology used to describe HF is based on left ventricular ejection fraction (LVEF). On the basis of ejection fraction (EF), HF comprises a wide range of patients, from those with normal LVEF(typically considered ase" $50 \%$ called HF with preserved EF,HFpEF) to those with reduced EF (typically considered as $<40 \%$ called HF with reduced EF, HFrEF). Patients with LVEF in the range of $40-49 \%$ represent a grey area which is now defined as HF with mildly reduced EF (HFmrEF). ${ }^{1}$

LVEF is such an important parameter that most clinical trials published after 1990 selected patients based on LVEF (usually measured using echocardiography). Thus differentiation of patients based on EF is important due to different underlying aetiologies, demographics, comorbidites and response to therapies.

Virtually all forms of acquired heart diseases may be associated with abnormalities of systolic function at some point in their natural history. LVEF is thecornerstone of risk evaluation and management in most cardiac diseases. This simplest and most widely used parameterprovides valuable prognostic information, plays a crucial role in selection of

Address of Correspondence: M Atahar Ali, Professor of Cardiology, National Institute of Cardiovascular Diseases (NICVD), Dhaka, Bangladesh. Cell: +8801711595770, Email: dha_042000@yahoo.com medical therapy as well as device based therapy and is instrumental in determining the timing of surgery for structural heart disease. ${ }^{2,3}$

The EF is considered important in HF, not only because of its prognostic importance (the lower the EF the poorer the survival)but also becausemost clinical trials selected patients based uponEF. ${ }^{4}$ Although different modalities of EF measurement used in different clinical trials, SCD-HeFT trial shows thatsurvival did not differ according to modality of EF assessment like radionuclide angiography, echocardiography or contrastangiograms. ${ }^{7}$ It helps to determine the best course of treatment like choice of anti-arrhythmic drugs, selection of patient for device therapy.

It is a predictor of sudden cardiac death (SCD). Patients with LVEF $<35 \%$ have high risk of SCD as they are prone to develop malignant arrhythmia. So to prevent SCD and to improve quality of life by implantation of implantable cardioverter defibrillator (ICD) or cardiac resynchronization therapy (CRT), LVEF is an important factor to select the patient. ${ }^{1}$ It is also astrong predictor ofpersistent LV dysfunction and a grave long-term prognostic factor following acute myocardial infarction(AMI) (EF $<40 \%$ raises 2.47 times the risk of death during the first year after $\mathrm{Ml}$ and nearly 2 times during the 5 -year follow-up). ${ }^{8}$

Severely decreased (<30\%) LVEF itself is an independent predictor of perioperative outcome and a long-term risk factor for death in patients with HF undergoing elevated-risk noncardiac surgery. Survival after surgery for those with a LVEF d" $29 \%$ is significantly worse than for those with a LVEF > $29 \%{ }^{5}$

In spite of wide use of EF, it has a number of important limitations. The usefulness of EF is sometimes undermined by its strict cut-offs and also by variability of measurement in multiple techniques as well as by inter-observer and intraobserver variability. The long-standing emphasis on EF is misguiding. EF is erroneouslyassumed to be a measure of myocardial contractility. ${ }^{6}$ 
Despite its limitations, quantifying EF is accepted by the cardiology community. The evidence-base for modern cardiology is so heavily based on this simple measurement that it is unlikely to disappear. The ubiquitous presence of cardiovascular diseases in currently-aging societies mandates an inexpensive, widely available test that is able to provide hemodynamic assessmentand it became the identity of every cardiac patient as the mobile number of individual person. So the wide use of EF suggests that more formal quality control, automation, and quantitation may be desirable. ${ }^{3}$

Estimation of prognosis for morbidity, disability and death helps patients, their families and clinicians to decide the appropriate type and timing of therapies. In recent decades several multivariate prognostic risk scores have been developed on the basis of EF for different populations of patients with HF and some are available as interactive online applications. Thus LVEF has become a social number and it is more important than mobile number to a heart failure patient. Not only for HF but also it is a cornerstone of risk evaluation and management in most cardiac diseases.

\section{References}

1. Ponikowski P, Voors AA, Anker SD, Bueno H, Cleland JGF, Coats AJS, et al. 2016 ESC Guidelines for the diagnosis and treatment of acute and chronic heart failure: The Task Force for the diagnosis and treatment of acute and chronic heart failure of the European Society of Cardiology (ESC)Developed with the special contribution of the Heart Failure Association (HFA) of the ESC. Eur Heart J. 2016;37:2129-200.

2. Armstrong WF, Ryan T, Feigenbaum H. Feigenbaum's echocardiography. 7th ed. Philadelphia, Pa., London: Lippincott Williams \& Wilkins. 2010.
3. Lang RM. ASE's comprehensive echocardiography. Philadelphia, Pennsylvania: Elsevier. 2016.

4. McMurray JJV, Adamopoulos S, Anker SD, Auricchio A, Böhm M, Dickstein K, et al. ESC Guidelines for the diagnosis and treatment of acute and chronic heart failure 2012: The Task Force for the Diagnosis and Treatment of Acute and Chronic Heart Failure 2012 of the European Society of Cardiology. Developed in collaboration with the Heart Failure Association (HFA) of the ESC. Eur Heart J. 2012;33:1787-847.

5. Fleisher LA, Fleischmann KE, Auerbach AD, Barnason SA, Beckman JA, Bozkurt B, et al. 2014 ACC/AHA guideline on perioperative cardiovascular evaluation and management of patients undergoing noncardiac surgery: A report of the American College of Cardiology/ American Heart Association Task Force on practice guidelines. J Am Coll Cardiol. 2014;64:e77-137.

6. Konstam MA, Abboud FM. Ejection Fraction: Misunderstood and Overrated (Changing the Paradigm in Categorizing Heart Failure). Circulation. 2017;135:717-9.

7. Gula LJ, Klein GJ, Hellkamp AS, Massel D, Krahn AD, Skanes AC, et al. Ejection fraction assessment and survival: an analysis of the Sudden Cardiac Death in Heart Failure Trial (SCD-HeFT). Am Heart J. 2008 Dec;156(6):1196-200.

8. Brooks GC, Lee BK, Rao R, Lin F, Morin DP, Zweibel SL, et al.; PREDICTS Investigators. Predicting Persistent Left Ventricular Dysfunction Following Myocardial Infarction: The PREDICTS Study. J Am Coll Cardiol. 2016 Mar 15;67(10):1186-1196.. 